\title{
NONEXISTENCE, EXISTENCE AND SYMMETRY OF NORMALIZED GROUND STATES TO CHOQUARD EQUATIONS WITH A LOCAL PERTURBATION
}

\author{
XINFU LI
}

ABStRaCt. We study the Choquard equation with a local perturbation

$$
-\Delta u=\lambda u+\left(I_{\alpha} *|u|^{p}\right)|u|^{p-2} u+\mu|u|^{q-2} u, x \in \mathbb{R}^{N}
$$

having prescribed mass

$$
\int_{\mathbb{R}^{N}}|u|^{2} d x=a^{2}
$$

For a $L^{2}$-critical or $L^{2}$-supercritical perturbation $\mu|u|^{q-2} u$, we prove nonexistence, existence and symmetry of normalized ground states, by using the mountain pass lemma, the Pohožaev constraint method, the Schwartz symmetrization rearrangements and some theories of polarizations. In particular, our results cover the Hardy-Littlewood-Sobolev upper critical exponent case $p=(N+\alpha) /(N-2)$. Our results are a nonlocal counterpart of the results in [18, 35, 37].

\section{INTRODUCTION AND MAIN RESULTS}

We consider the equation

$$
-\Delta u=\lambda u+\left(I_{\alpha} *|u|^{p}\right)|u|^{p-2} u+\mu|u|^{q-2} u, x \in \mathbb{R}^{N},
$$

where $N \geq 1, \alpha \in(0, N), I_{\alpha}$ is the Riesz potential defined for every $x \in \mathbb{R}^{N} \backslash\{0\}$ by

$$
I_{\alpha}(x)=\frac{A_{\alpha}(N)}{|x|^{N-\alpha}}, A_{\alpha}(N)=\frac{\Gamma\left(\frac{N-\alpha}{2}\right)}{\Gamma\left(\frac{\alpha}{2}\right) \pi^{N / 2} 2^{\alpha}}
$$

with $\Gamma$ denoting the Gamma function (see [32, P.19), $p$ and $q$ will be defined later. The equation (1.1) is usually called the nonlinear Choquard equation. For the physical case $N=3, p=2, \alpha=2$ and $\mu=0$, (1.1) was investigated by Pekar in [30] to study the quantum theory of a polaron at rest. In [22, Choquard applied it as an approximation to Hartree-Fock theory of one component plasma. It also arises in multiple particles systems [13] and quantum mechanics [31].

When looking for solutions to (1.1), a possible choice is to fix $\lambda<0$ and to search for solutions to (1.1) as critical points of the action functional

$$
J(u):=\int_{\mathbb{R}^{N}}\left(\frac{1}{2}|\nabla u|^{2}-\frac{\lambda}{2}|u|^{2}-\frac{1}{2 p}\left(I_{\alpha} *|u|^{p}\right)|u|^{p}-\frac{\mu}{q}|u|^{q}\right),
$$

see for example [19, 20, 26, 29] and the references therein.

2020 Mathematics Subject Classification. 35J20, 35B06, 35B33.

Key words and phrases. Normalized ground state; symmetry; Choquard equation; upper critical exponent. 
Alternatively, from a physical point of view, it is interesting to find solutions of (1.1) having prescribed mass

$$
\int_{\mathbb{R}^{N}}|u|^{2}=a^{2} .
$$

In this direction, define on $H^{1}\left(\mathbb{R}^{N}, \mathbb{C}\right)$ the energy functional

$$
E(u):=\frac{1}{2} \int_{\mathbb{R}^{N}}|\nabla u|^{2}-\frac{1}{2 p} \int_{\mathbb{R}^{N}}\left(I_{\alpha} *|u|^{p}\right)|u|^{p}-\frac{\mu}{q} \int_{\mathbb{R}^{N}}|u|^{q} .
$$

It is standard to check that $E \in C^{1}$ under some assumptions on $p$ and $q$ and a critical point of $E$ constrained to

$$
S_{a}:=\left\{u \in H^{1}\left(\mathbb{R}^{N}, \mathbb{C}\right): \int_{\mathbb{R}^{N}}|u|^{2}=a^{2}\right\}
$$

gives rise to a solution to (1.1), satisfying (1.3). Such solution is usually called a normalized solution of (1.1). In this method, the parameter $\lambda \in \mathbb{R}$ arises as a Lagrange multiplier, which depends on the solution and is not a priori given. In this paper, we will focus on the normalized ground state of (1.1), defined as follows:

Definition 1.1. We say that $u$ is a normalized ground state to (1.1) on $S_{a}$ if

$$
E(u)=c^{g}:=\inf \left\{E(v): v \in S_{a},\left(\left.E\right|_{S_{a}}\right)^{\prime}(v)=0\right\} .
$$

The set of the normalized ground states will be denoted by $\mathcal{G}$.

When studying normalized solutions of the Choquard equation, three exponents play an important role: the Hardy-Littlewood-Sobolev upper critical exponent $\bar{p}$, the Hardy-Littlewood-Sobolev lower critical exponent $\underline{p}$ and the $L^{2}$-critical exponent $p^{*}$ defined by

$$
\bar{p}:= \begin{cases}\infty, & N=1,2, \\ \frac{N+\alpha}{N-2}, & N \geq 3, \quad \underline{p}:=\frac{N+\alpha}{N}, \quad p^{*}:=1+\frac{2+\alpha}{N} .\end{cases}
$$

Recently, researchers pay much attention to the normalized solutions to the Choquard equation (1.1) for the case $\mu=0$, i.e.,

$$
-\Delta u=\lambda u+\left(I_{\alpha} *|u|^{p}\right)|u|^{p-2} u, x \in \mathbb{R}^{N} .
$$

For $\underline{p}<p<p^{*}$, Ye [41] obtained a normalized ground state to (1.4) by considering the minimizer of $E$ constrained on $S_{a}$. For $p^{*}<p<\bar{p}$, the functional $E$ is no longer bounded from below on $S_{a}$, Luo [25] obtained a normalized ground state to (1.4) by considering the minimizer of $E$ constrained on $\mathcal{P}$ defined as in (1.9). For $p=p^{*}$, by scaling invariance, the result is delicate, see [7] and [41. In the case $N \geq 3, \mathrm{Li}$ and Ye in 21] considered the general equation

$$
-\Delta u=\lambda u+\left(I_{\alpha} * F(u)\right) f(u), x \in \mathbb{R}^{N}
$$

under a set of assumptions on $f$, which when $f$ takes the special form $f(s)=$ $C_{1}|s|^{r-2} s+C_{2}|s|^{p-2} s$ requires that $p^{*}<r \leq p<\bar{p}$. Bartsch, Liu and Liu [1] further considered the existence of a normalized ground state and the existence of infinitely many normalized solutions to (1.5) in all dimensions $N \geq 1$. Recently, Yuan, Chen and Tang [42] reconsidered (1.5) with more general $f \in C(\mathbb{R}, \mathbb{R})$. When $p=p^{*}$ and $2<q<q^{*}$, 24] considered the existence and orbital stability of the normalized ground state to (1.1). Most existing results considered similar equations to (1.1) with one positive nonlinearity and one negative nonlinearity, see [3, 4, 8, 33. for the study of the Schrödinger-Poisson system. 
To our knowledge, there are no papers considering the normalized solutions to the Choquard equation with the Hardy-Littlewood-Sobolev upper critical exponent $p=\bar{p}$. By [27, for fixed $\lambda<0$, (1.4) has no solutions in $H^{1}\left(\mathbb{R}^{N}\right)$ under the range $p \geq \bar{p}$. However, the equation

$$
-\Delta u=\left(I_{\alpha} *|u|^{\bar{p}}\right)|u|^{\bar{p}-2} u, x \in \mathbb{R}^{N}
$$

has solutions in $D^{1,2}\left(\mathbb{R}^{N}\right)$, see [11]. So it is interesting to study the existence of normalized solutions to (1.4) with $p=\bar{p}$ under a local perturbation $\mu|u|^{q-2} u$, namely equation (1.1). In this paper, we will give an affirmative answer to the problem.

Now, we present our first main result.

Theorem 1.2. Assume $N \geq 1, \alpha \in(0, N), a>0, \mu>0, q^{*}=2+4 / N$,

$$
q^{*} \leq q<2^{*}:=\left\{\begin{array}{ll}
\infty, & N=1,2, \\
\frac{2 N}{N-2}, & N \geq 3,
\end{array} p^{*}<p \begin{cases}<\bar{p}, & N=1,2, \\
\leq \bar{p}, & N \geq 3 .\end{cases}\right.
$$

If $q=q^{*}$, we further assume that $\mu a^{4 / N}<\left(a_{N}^{*}\right)^{4 / N}$, where $a_{N}^{*}$ is defined in (2.1). Then the equation (1.1) has a mountain pass type normalized ground state, $c^{g}>0$ if $p<\bar{p}$, and

$$
0<c^{g}<\frac{2+\alpha}{2(N+\alpha)} S_{\alpha}^{\frac{N+\alpha}{2+\alpha}}
$$

if $p=\bar{p}$, where $S_{\alpha}$ is defined in (3.7). Moreover, every $u \in \mathcal{G}$ solves (1.1) with some $\lambda=\lambda(u)<0$.

Remark 1.3. Recently, Yang [40] considered the fractional equation

$$
(-\Delta)^{\sigma} u=\lambda u+|u|^{q-2} u+\mu\left(I_{\alpha} *|u|^{p}\right)|u|^{p-2} u, x \in \mathbb{R}^{N}
$$

with $N \geq 2, \sigma \in(0,1)$ and $\alpha \in(N-2 \sigma, N)$. Under the assumptions

$$
2+\frac{4 \sigma}{N}<q<\frac{2 N}{N-2 \sigma} \text { and } 1+\frac{2 \sigma+\alpha}{N} \leq p \leq \frac{q}{2}+\frac{\alpha}{N},
$$

they obtained a mountain pass type positive radial normalized ground state to (1.7). Note that the case $p>\frac{q}{2}+\frac{\alpha}{N}$ was left in [40]. In this paper, by using the Schwartz symmetrization rearrangements, we can show that $c_{r}^{m p}=c^{m p}=c^{p o}$ and then complement the interval, see the proof of Theorem 1.2.

Our second main result is about the positivity and radial symmetry of the normalized ground states.

Theorem 1.4. Assume the conditions in Theorem 1.2 hold. Let $u$ be a normalized ground state to (1.1) on $S_{a}$, then

(1) $|u|>0$ is a normalized ground state to (1.1);

(2) there exist $x_{0} \in \mathbb{R}^{N}$ and a non-increasing positive function $v:(0, \infty) \rightarrow \mathbb{R}$ such that $|u|=v\left(\left|x-x_{0}\right|\right)$ for almost every $x \in \mathbb{R}^{N}$;

(3) $u=e^{i \theta}|u|$ for some $\theta \in \mathbb{R}$.

By using the methods used in [18, we can obtain the following nonexistence result, see [37] for a different proof.

Theorem 1.5. Let $N \geq 1, \alpha \in(0, N), a>0, \mu>0, q=q^{*}=2+4 / N$,

$$
p^{*}<p \begin{cases}<\bar{p}, \quad N=1,2 \\ \leq \bar{p}, \quad N \geq 3\end{cases}
$$


and $\mu a^{4 / N} \geq\left(a_{N}^{*}\right)^{4 / N}$ with $a_{N}^{*}$ defined in (2.1). Then $c^{p o}=0$ and thus $c^{p o}$ can not be attained and (1.1) has no normalized ground states, where $c^{p o}$ is defined in (1.9).

Remark 1.6. The proof of Theorem 1.5 can be done by modifying the proof of Theorem 1.5 in [18] done to the Schrödinger equation, so we omit the proof here. The most difference is in Case 2 of Lemma 3.2 in [18] choosing $\tilde{u}$ such that $\|\tilde{u}\|_{2}^{2}=$ $a^{2}$ and $\|\tilde{u}\|_{q}^{q}=\left(\int_{\mathbb{R}^{N}}\left(I_{\alpha} *|\tilde{u}|^{p}\right)|\tilde{u}|^{p}\right)^{\frac{1}{p \eta_{p}}}$, where $\eta_{p}$ is defined in (1.8).

Now we outline the methods used in this paper to prove Theorems 1.2 and 1.4 For the interaction of $\left(I_{\alpha} *|u|^{p}\right)|u|^{p-2} u$ and $|u|^{q-2} u$, and the inequality for the Schwartz symmetrization rearrangement

$$
\int_{\mathbb{R}^{N}}\left(I_{\alpha} *\left(|u|^{*}\right)^{p}\right)\left(|u|^{*}\right)^{p} \geq \int_{\mathbb{R}^{N}}\left(I_{\alpha} *|u|^{p}\right)|u|^{p}
$$

where $|u|^{*}$ is the Schwartz symmetrization rearrangement of $|u|$, the methods used in [3, 25] or 34] can not solve our problems for the optimal range of parameters. In this paper, we combine the methods used in [17, 28 (see also [16, 20]) and [34] to prove Theorems 1.2 and 1.4. Using this method, we can treat the existence and symmetry of the normalized ground states to (1.1) simultaneously. Precisely, we first use the mountain pass lemma to obtain a Palais-Smale sequence $\left\{u_{n}\right\}$ of $E$ on $S_{a} \cap H_{r}^{1}\left(\mathbb{R}^{N}\right)$ with $P\left(u_{n}\right) \rightarrow 0$ and $E\left(u_{n}\right) \rightarrow c_{r}^{m p}$ as $n \rightarrow \infty$, where

and

$$
P(u):=\int_{\mathbb{R}^{N}}|\nabla u|^{2}-\eta_{p} \int_{\mathbb{R}^{N}}\left(I_{\alpha} *|u|^{p}\right)|u|^{p}-\mu \gamma_{q} \int_{\mathbb{R}^{N}}|u|^{q}
$$

$$
\eta_{p}:=\frac{N}{2}-\frac{N+\alpha}{2 p}, \quad \gamma_{q}:=\frac{N}{2}-\frac{N}{q}
$$

Secondly, by using the Pohožaev constraint method and the Schwartz symmetrization rearrangements, we can show that $c_{r}^{m p}=c^{m p}=c^{p o}$, where

$$
c^{p o}:=\inf _{u \in \mathcal{P}} E(u), \quad \mathcal{P}:=\left\{u \in S_{a}: P(u)=0\right\} .
$$

Thirdly, by using the radial symmetry of $\left\{u_{n}\right\}$, the bounds of $c^{p o}$, and the relationship of $c^{p o}$ and $c^{g}$, we can show that $\left\{u_{n}\right\}$ converges to a normalized ground state of (1.1). Lastly, by associating any normalized ground state $u$ to a special path in $\Gamma$, and using $c^{m p}=c^{p o}$ and the theories of polarizations, we can obtain the radial symmetry of $|u|$.

This paper is organized as follows. In Section 2, we cite some preliminaries. Sections 3 and 4 are devoted to the proof of Theorems 1.2 and 1.4 respectively.

Notation: In this paper, it is understood that all functions, unless otherwise stated, are complex valued, but for simplicity we write $L^{t}\left(\mathbb{R}^{N}\right), H^{1}\left(\mathbb{R}^{N}\right), D^{1,2}\left(\mathbb{R}^{N}\right)$, .... For $1 \leq t<\infty, L^{t}\left(\mathbb{R}^{N}\right)$ is the usual Lebesgue space endowed with the norm $\|u\|_{t}^{t}:=\int_{\mathbb{R}^{N}}|u|^{t}, H^{1}\left(\mathbb{R}^{N}\right)$ is the usual Sobolev space endowed with the norm

$$
\|u\|^{2}:=\int_{\mathbb{R}^{N}}\left(|\nabla u|^{2}+|u|^{2}\right),
$$

and $D^{1,2}\left(\mathbb{R}^{N}\right):=\left\{u \in L^{2^{*}}\left(\mathbb{R}^{N}\right):|\nabla u| \in L^{2}\left(\mathbb{R}^{N}\right)\right\} . H_{r}^{1}\left(\mathbb{R}^{N}\right)$ denotes the subspace of functions in $H^{1}\left(\mathbb{R}^{N}\right)$ which are radially symmetric with respect to zero. $S_{a, r}:=$ $S_{a} \cap H_{r}^{1}\left(\mathbb{R}^{N}\right) . C, C_{1}, C_{2}, \ldots$ denote positive constants, whose values can change from line to line. 


\section{Preliminaries}

The following Gagliardo-Nirenberg inequality can be found in [38].

Lemma 2.1. Let $N \geq 1$ and $2<p<2^{*}$, then the following sharp GagliardoNirenberg inequality

$$
\|u\|_{p} \leq C_{N, p}\|u\|_{2}^{1-\gamma_{p}}\|\nabla u\|_{2}^{\gamma_{p}}
$$

holds for any $u \in H^{1}\left(\mathbb{R}^{N}\right)$, where the sharp constant $C_{N, p}$ is

$$
C_{N, p}^{p}=\frac{2 p}{2 N+(2-N) p}\left(\frac{2 N+(2-N) p}{N(p-2)}\right)^{\frac{N(p-2)}{4}} \frac{1}{\left\|Q_{p}\right\|_{2}^{p-2}}
$$

and $Q_{p}$ is the unique positive radial solution of equation

$$
-\Delta Q+Q=|Q|^{p-2} Q .
$$

In the special case $p=2+4 / N, C_{N, p}^{p}=p /\left(2\left\|Q_{p}\right\|_{2}^{4 / N}\right)$, or equivalently,

$$
\left\|Q_{p}\right\|_{2}=\left(\frac{p}{2 C_{N, p}^{p}}\right)^{N / 4}=: a_{N}^{*}
$$

The following well-known Hardy-Littlewood-Sobolev inequality can be found in 23.

Lemma 2.2. Let $N \geq 1, p, r>1$ and $0<\beta<N$ with $1 / p+(N-\beta) / N+1 / r=2$. Let $u \in L^{p}\left(\mathbb{R}^{N}\right)$ and $v \in L^{r}\left(\mathbb{R}^{N}\right)$. Then there exists a sharp constant $C(N, \beta, p)$, independent of $u$ and $v$, such that

$$
\left|\int_{\mathbb{R}^{N}} \int_{\mathbb{R}^{N}} \frac{u(x) v(y)}{|x-y|^{N-\beta}} d x d y\right| \leq C(N, \beta, p)\|u\|_{p}\|v\|_{r} .
$$

If $p=r=\frac{2 N}{N+\beta}$, then

$$
C(N, \beta, p)=C_{\beta}(N)=\pi^{\frac{N-\beta}{2}} \frac{\Gamma\left(\frac{\beta}{2}\right)}{\Gamma\left(\frac{N+\beta}{2}\right)}\left\{\frac{\Gamma\left(\frac{N}{2}\right)}{\Gamma(N)}\right\}^{-\frac{\beta}{N}} .
$$

Remark 2.3. (1). By the Hardy-Littlewood-Sobolev inequality above, for any $v \in$ $L^{s}\left(\mathbb{R}^{N}\right)$ with $s \in(1, N / \alpha), I_{\alpha} * v \in L^{\frac{N s}{N-\alpha s}}\left(\mathbb{R}^{N}\right)$ and

$$
\left\|I_{\alpha} * v\right\|_{L^{\frac{N s}{N-\alpha s}}} \leq C\|v\|_{L^{s}}
$$

where $C>0$ is a constant depending only on $N, \alpha$ and $s$.

(2). By the Hardy-Littlewood-Sobolev inequality above and the Sobolev embedding theorem, we obtain

$$
\int_{\mathbb{R}^{N}}\left(I_{\beta} *|u|^{p}\right)|u|^{p} \leq C\left(\int_{\mathbb{R}^{N}}|u|^{\frac{2 N p}{N+\beta}}\right)^{1+\beta / N} \leq C\|u\|_{H^{1}\left(\mathbb{R}^{N}\right)}^{2 p}
$$

for any $p \in[1+\beta / N,(N+\beta) /(N-2)]$ if $N \geq 3$ and $p \in[1+\beta / N,+\infty)$ if $N=$ 1,2 , where $C>0$ is a constant depending only on $N, \beta$ and $p$.

The following Gagliardo-Nirenberg inequality for the convolution problem can be found in [9] and 27. 
Lemma 2.4. Let $N \geq 1,0<\beta<N, 1+\beta / N<p<\infty$ if $N=1,2$, and $1+\beta / N<p<(N+\beta) /(N-2)$ if $N \geq 3$, then

$$
\left(\int_{\mathbb{R}^{N}}\left(I_{\beta} *|u|^{p}\right)|u|^{p}\right)^{\frac{1}{2 p}} \leq C_{\beta, p}\|\nabla u\|_{2}^{\eta_{p}}\|u\|_{2}^{1-\eta_{p}} .
$$

The best constant $C_{\beta, p}$ is defined by

$$
\left(C_{\beta, p}\right)^{2 p}=\frac{2 p}{2 p-N p+N+\beta}\left(\frac{2 p-N p+N+\beta}{N p-N-\beta}\right)^{(N p-N-\beta) / 2}\left\|W_{p}\right\|_{2}^{2-2 p},
$$

where $W_{p}$ is a radially ground state solution of the elliptic equation

$$
-\Delta W+W=\left(I_{\beta} *|W|^{p}\right)|W|^{p-2} W
$$

In particular, in the $L^{2}$-critical case, i.e., $p=1+(2+\beta) / N,\left(C_{\beta, p}\right)^{2 p}=p\left\|W_{p}\right\|_{2}^{2-2 p}$.

Remark 2.5. Note that $W_{p}$ may be not unique, but it has the same $L^{2}$-norm, see 9]. Hence, if we define $R_{p}=\left\|W_{p}\right\|_{2}$, then $R_{p}$ is a constant.

The following fact is used in this paper (see [20, 28]). For the readers' convenience, we give the proof here.

Lemma 2.6. Assume that $N \geq 1, \alpha \in(0, N), 1+\alpha / N \leq p<\infty$ if $N=1,2$, and $1+\alpha / N \leq p \leq(N+\alpha) /(N-2)$ if $N \geq 3$. Let $\left\{u_{k}\right\} \subset H^{1}\left(\mathbb{R}^{N}\right)$ be a sequence satisfying that $u_{k} \rightarrow u$ weakly in $H^{1}\left(\mathbb{R}^{N}\right)$. Then, for any $\varphi \in H^{1}\left(\mathbb{R}^{N}\right)$,

$$
\int_{\mathbb{R}^{N}}\left(I_{\alpha} *\left|u_{k}\right|^{p}\right)\left|u_{k}\right|^{p-2} u_{k} \varphi \rightarrow \int_{\mathbb{R}^{N}}\left(I_{\alpha} *|u|^{p}\right)|u|^{p-2} u \varphi
$$

as $k \rightarrow \infty$.

Proof. Up to a subsequence, $\left\{u_{k}\right\}$ is bounded in $H^{1}\left(\mathbb{R}^{N}\right), u_{k} \rightarrow u$ weakly in $H^{1}\left(\mathbb{R}^{N}\right)$ and $u_{k} \rightarrow u$ a.e. in $\mathbb{R}^{N}$. By the Sobolev embedding theorem, $\left\{u_{k}\right\}$ is bounded in $L^{2}\left(\mathbb{R}^{N}\right) \cap L^{2^{*}}\left(\mathbb{R}^{N}\right)$. Therefore, the sequence $\left\{\left|u_{k}\right|^{p}\right\}$ is bounded in $L^{\frac{2 N}{N+\alpha}}\left(\mathbb{R}^{N}\right)$, and then

$$
\left|u_{k}\right|^{p} \rightarrow|u|^{p} \text { weakly in } L^{\frac{2 N}{N+\alpha}}\left(\mathbb{R}^{N}\right) .
$$

By the Rellich theorem, $u_{k} \rightarrow u$ strongly in $L_{\mathrm{loc}}^{r}\left(\mathbb{R}^{N}\right)$ for $r \in\left[1,2^{*}\right)$ and then $\left|u_{k}\right|^{p-2} u_{k} \rightarrow|u|^{p-2} u$ strongly in $L_{\text {loc }}^{\frac{2 N p \delta}{(p-1)(N+\alpha)}}\left(\mathbb{R}^{N}\right)$ with $\delta \in((N+\alpha) /(2 N), 1)$ (see Theorem A.2 in 39]). Hence, $\left|u_{k}\right|^{p-2} u_{k} \varphi \rightarrow|u|^{p-2} u \varphi$ strongly in $L^{\frac{2 N}{N+\alpha}}\left(\mathbb{R}^{N}\right)$ for any $\varphi \in C_{c}^{\infty}\left(\mathbb{R}^{N}\right)$. By Remark 2.3 we have

$$
I_{\alpha} *\left(\left|u_{k}\right|^{p-2} u_{k} \varphi\right) \rightarrow I_{\alpha} *\left(|u|^{p-2} u \varphi\right)
$$

strongly in $L^{\frac{2 N}{N-\alpha}}\left(\mathbb{R}^{N}\right)$. Thus,

$$
\begin{aligned}
& \int_{\mathbb{R}^{N}}\left(I_{\alpha} *\left|u_{k}\right|^{p}\right)\left|u_{k}\right|^{p-2} u_{k} \varphi-\int_{\mathbb{R}^{N}}\left(I_{\alpha} *|u|^{p}\right)|u|^{p-2} u \varphi \\
= & \int_{\mathbb{R}^{N}}\left|u_{k}\right|^{p}\left(I_{\alpha} *\left(\left|u_{k}\right|^{p-2} u_{k} \varphi\right)\right)-\int_{\mathbb{R}^{N}}|u|^{p}\left(I_{\alpha} *\left(|u|^{p-2} u \varphi\right)\right) \\
= & \int_{\mathbb{R}^{N}}\left|u_{k}\right|^{p}\left(I_{\alpha} *\left(\left|u_{k}\right|^{p-2} u_{k} \varphi\right)-I_{\alpha} *\left(|u|^{p-2} u \varphi\right)\right) \\
& \quad+\int_{\mathbb{R}^{N}}\left(\left|u_{k}\right|^{p}-|u|^{p}\right)\left(I_{\alpha} *\left(|u|^{p-2} u \varphi\right)\right) \\
\rightarrow 0 &
\end{aligned}
$$


as $k \rightarrow \infty$. Since $C_{c}^{\infty}\left(\mathbb{R}^{N}\right)$ is dense in $H^{1}\left(\mathbb{R}^{N}\right)$, the proof is complete.

The following Pohožaev identity is cited from [19], where the proof is given for $N \geq 3$ and $\lambda>0$ but it clearly extends to $N=1,2$ and $\lambda \in \mathbb{R}$.

Lemma 2.7. Let $N \geq 1, \alpha \in(0, N), \lambda \in \mathbb{R}, \mu \in \mathbb{R}, p \in[1+\alpha / N,+\infty)$ and $q \in[2,+\infty)$ for $N=1,2, p \in[1+\alpha / N,(N+\alpha) /(N-2)]$ and $q \in[2,2 N /(N-2)]$ for $N \geq 3$. If $u \in H^{1}\left(\mathbb{R}^{N}\right)$ is a solution to (1.1), then $u$ satisfies the Pohožaev identity

$$
\frac{N-2}{2} \int_{\mathbb{R}^{N}}|\nabla u|^{2}=\frac{N \lambda}{2} \int_{\mathbb{R}^{N}}|u|^{2}+\frac{N+\alpha}{2 p} \int_{\mathbb{R}^{N}}\left(I_{\alpha} *|u|^{p}\right)|u|^{p}+\frac{\mu N}{q} \int_{\mathbb{R}^{N}}|u|^{q} .
$$

Lemma 2.8. Assume the conditions in Lemma 2.7 hold. If $u \in H^{1}\left(\mathbb{R}^{N}\right)$ is a solution to (1.1), then $P(u)=0$.

Proof. Multiplying (1.1) by $u$ and integrating over $\mathbb{R}^{N}$, we derive

$$
\int_{\mathbb{R}^{N}}|\nabla u|^{2}=\lambda \int_{\mathbb{R}^{N}}|u|^{2}+\int_{\mathbb{R}^{N}}\left(I_{\alpha} *|u|^{p}\right)|u|^{p}+\mu \int_{\mathbb{R}^{N}}|u|^{q},
$$

which combines with the Pohožaev identity from Lemma 2.7 gives that $P(u)=$ 0 .

\section{Proof of Theorem 1.2}

In this section, we first study the properties of $c^{p o}$ defined in (1.9), and then give the proof of Theorem 1.2 ,

For $u \in S_{a}$ and $s \in \mathbb{R}$, define

$$
(s \star u)(x):=e^{\frac{N}{2} s} u\left(e^{s} x\right), x \in \mathbb{R}^{N} .
$$

Then $s \star u \in S_{a}$. Consider the fiber maps

$$
\begin{gathered}
\Psi_{u}(s):=E(s \star u)=\frac{1}{2} e^{2 s} \int_{\mathbb{R}^{N}}|\nabla u|^{2}-\frac{1}{2 p} e^{(N p-N-\alpha) s} \int_{\mathbb{R}^{N}}\left(I_{\alpha} *|u|^{p}\right)|u|^{p} \\
-\frac{\mu}{q} e^{\left(\frac{N}{2} q-N\right) s} \int_{\mathbb{R}^{N}}|u|^{q}
\end{gathered}
$$

and

$$
\begin{gathered}
P(s \star u)=e^{2 s} \int_{\mathbb{R}^{N}}|\nabla u|^{2}-\eta_{p} e^{(N p-N-\alpha) s} \int_{\mathbb{R}^{N}}\left(I_{\alpha} *|u|^{p}\right)|u|^{p} \\
-\mu \gamma_{q} e^{\left(\frac{N}{2} q-N\right) s} \int_{\mathbb{R}^{N}}|u|^{q} .
\end{gathered}
$$

We have the following lemma.

Lemma 3.1. Assume the conditions in Theorem 1.2 hold. Then for every $u \in S_{a}$, there exists a unique $s_{u} \in \mathbb{R}$ such that $P\left(s_{u} \star u\right)=0 . s_{u}$ is the unique critical point of the function $\Psi_{u}$, and is a strict maximum point at positive level. Moreover, $P(u) \leq 0$ is equivalent to $s_{u} \leq 0$.

Proof. Set $P(s \star u)=e^{2 s} g_{u}(s)$, where

$$
\begin{gathered}
g_{u}(s)=\int_{\mathbb{R}^{N}}|\nabla u|^{2}-\eta_{p} e^{(N p-N-\alpha-2) s} \int_{\mathbb{R}^{N}}\left(I_{\alpha} *|u|^{p}\right)|u|^{p} \\
-\mu \gamma_{q} e^{\left(\frac{N}{2} q-N-2\right) s} \int_{\mathbb{R}^{N}}|u|^{q} .
\end{gathered}
$$


If $q>q^{*}$, we have $\frac{N}{2} q-N-2>0$. If $q=q^{*}$ and $\mu a^{4 / N}<\left(a_{N}^{*}\right)^{4 / N}$, we have $\frac{N}{2} q-N-2=0$ and by the Gagliardo-Nirenberg inequality (Lemma 2.1),

$$
\mu \gamma_{q} \int_{\mathbb{R}^{N}}|u|^{q} d x \leq \mu \gamma_{q} C_{N, q}^{q} a^{q\left(1-\gamma_{q}\right)}\|\nabla u\|_{2}^{2}<\|\nabla u\|_{2}^{2}
$$

Since $N p-N-\alpha-2>0$, so in both cases, $g_{u}(s)>0$ for $s \ll 0, g_{u}(s)<0$ for $s \gg 0$, and $g_{u}^{\prime}(s)<0$ for $s \in \mathbb{R}$. Thus, $g_{u}(s)$ has a unique zero $s_{u}$ as well as $P(s \star u)$. It is obvious that $P(u) \leq 0 \Leftrightarrow s_{u} \leq 0$.

By direct calculations, we have $\Psi_{u}^{\prime}(s)=P(s \star u), \lim _{s \rightarrow-\infty} \Psi_{u}(s)=0, \Psi_{u}(s)>0$ for $s \ll 0$ and $\lim _{s \rightarrow+\infty} \Psi_{u}(s)=-\infty$. Thus, $s_{u}$ is the unique critical point of $\Psi_{u}(s)$ and $\Psi_{u}\left(s_{u}\right)=\max _{s \in \mathbb{R}} \Psi_{u}(s)>0$.

Lemma 3.2. Assume the conditions in Theorem 1.2 hold. Then $c^{p o}>0$.

Proof. By Lemma 3.1, $\mathcal{P} \neq \emptyset$.

Case $\mathbf{1}(p \neq \bar{p})$. For any $u \in \mathcal{P}$, by the Gagliardo-Nirenberg inequality (Lemmas 2.1 and 2.4), we have

$$
\begin{aligned}
\int_{\mathbb{R}^{N}}|\nabla u|^{2} & =\eta_{p} \int_{\mathbb{R}^{N}}\left(I_{\alpha} *|u|^{p}\right)|u|^{p}+\mu \gamma_{q} \int_{\mathbb{R}^{N}}|u|^{q} \\
& \leq \eta_{p} C_{\alpha, p}^{2 p}\|u\|_{2}^{2 p\left(1-\eta_{p}\right)}\|\nabla u\|_{2}^{2 p \eta_{p}}+\mu \gamma_{q} C_{N, q}^{q}\|u\|_{2}^{q\left(1-\gamma_{q}\right)}\|\nabla u\|_{2}^{q \gamma_{q}} \\
& =\mu \gamma_{q} C_{N, q}^{q} a^{q\left(1-\gamma_{q}\right)}\|\nabla u\|_{2}^{q \gamma_{q}}+\eta_{p} C_{\alpha, p}^{2 p} a^{2 p\left(1-\eta_{p}\right)}\|\nabla u\|_{2}^{2 p \eta_{p}} .
\end{aligned}
$$

If $q>q^{*}$, then $q \gamma_{q}>2$. Since $2 p \eta_{p}>2$, (3.3) implies that there exists a constant $C>0$ such that $\|\nabla u\|_{2}^{2} \geq C$. Consequently,

$$
\eta_{p} \int_{\mathbb{R}^{N}}\left(I_{\alpha} *|u|^{p}\right)|u|^{p}+\mu \gamma_{q} \int_{\mathbb{R}^{N}}|u|^{q} \geq C
$$

If $q=q^{*}$ and $\mu a^{4 / N}<\left(a_{N}^{*}\right)^{4 / N}$, then $q \gamma_{q}=2$ and $\mu \gamma_{q} C_{N, q}^{q} a^{q\left(1-\gamma_{q}\right)}<1$. Since $2 p \eta_{p}>2$, 3.3. implies that there exists a constant $C>0$ such that $\|\nabla u\|_{2}^{2} \geq C$. Thus, it follows from (3.3) that

$$
\begin{aligned}
\eta_{p} \int_{\mathbb{R}^{N}}\left(I_{\alpha} *|u|^{p}\right)|u|^{p} & \geq\left(1-\mu \gamma_{q} C_{N, q}^{q} a^{q\left(1-\gamma_{q}\right)}\right)\|\nabla u\|_{2}^{2} \\
& \geq C\left(1-\mu \gamma_{q} C_{N, q}^{q} a^{q\left(1-\gamma_{q}\right)}\right) .
\end{aligned}
$$

Any way, there always exists $C_{1}>0$ such that for any $u \in \mathcal{P}$,

$$
E(u)=\left(\frac{\eta_{p}}{2}-\frac{1}{2 p}\right) \int_{\mathbb{R}^{N}}\left(I_{\alpha} *|u|^{p}\right)|u|^{p}+\left(\frac{\gamma_{q}}{2}-\frac{1}{q}\right) \mu \int_{\mathbb{R}^{N}}|u|^{q} \geq C_{1},
$$

which implies $c^{p o}>0$.

Case $2(p=\bar{p})$. Similarly to Case 1 , just in (3.3), we estimate the term $\int_{\mathbb{R}^{N}}\left(I_{\alpha} *\right.$ $\left.|u|^{\bar{p}}\right)|u|^{\bar{p}}$ by using (3.7), i.e.,

$$
\int_{\mathbb{R}^{N}}\left(I_{\alpha} *|u|^{\bar{p}}\right)|u|^{\bar{p}} \leq\left(\frac{\int_{\mathbb{R}^{N}}|\nabla u|^{2}}{S_{\alpha}}\right)^{\bar{p}} .
$$

Lemma 3.3. Assume the conditions in Theorem 1.2 hold. Then there exists $k>0$ sufficiently small such that

$$
0<\sup _{A_{k}} E<c^{p o} \text { and } u \in \overline{A_{k}} \Rightarrow E(u), P(u)>0
$$


where $A_{k}=\left\{u \in S_{a}:\|\nabla u\|_{2}^{2}<k\right\}$.

Proof. Case $1(p \neq \bar{p})$. By the Gagliardo-Nirenberg inequality, we have

$$
\begin{aligned}
& E(u) \geq \frac{1}{2}\|\nabla u\|_{2}^{2}-\frac{\mu}{q} C_{N, q}^{q} a^{q\left(1-\gamma_{q}\right)}\|\nabla u\|_{2}^{q \gamma_{q}}-\frac{1}{2 p} C_{\alpha, p}^{2 p} a^{2 p\left(1-\eta_{p}\right)}\|\nabla u\|_{2}^{2 p \eta_{p}}>0, \\
& P(u) \geq\|\nabla u\|_{2}^{2}-\mu \gamma_{q} C_{N, q}^{q} a^{q\left(1-\gamma_{q}\right)}\|\nabla u\|_{2}^{q \gamma_{q}}-\eta_{p} C_{\alpha, p}^{2 p} a^{2 p\left(1-\eta_{p}\right)}\|\nabla u\|_{2}^{2 p \eta_{p}}>0,
\end{aligned}
$$

if $u \in \overline{A_{k}}$ with $k$ small enough, see the proof of Lemma 3.2 for more details. If necessary replacing $k$ with a smaller quantity, recalling that $c^{p o}>0$ by Lemma 3.2 we also have

$$
E(u) \leq \frac{1}{2}\|\nabla u\|_{2}^{2}<c^{p o} .
$$

Case $2(p=\bar{p})$. Similarly to Case 1 , just in (3.6) $)$, we estimate the term $\int_{\mathbb{R}^{N}}\left(I_{\alpha} *\right.$ $\left.|u|^{\bar{p}}\right)|u|^{\bar{p}}$ by using (3.5).

Lemma 3.4. Let $N \geq 3, \alpha \in(0, N), a>0, \mu>0, p=\bar{p}$ and $q^{*} \leq q<2^{*}$. If $q=q^{*}$, we further assume that $\mu a^{4 / N}<\left(a_{N}^{*}\right)^{4 / N}$. Then

$$
c^{p o}<\frac{2+\alpha}{2(N+\alpha)} S_{\alpha}^{\frac{N+\alpha}{2+\alpha}} .
$$

Proof. For any $\epsilon>0$, we define

$$
u_{\epsilon}(x)=\varphi(x) U_{\epsilon}(x),
$$

where $\varphi(x) \in C_{c}^{\infty}\left(\mathbb{R}^{N}\right)$ is a cut off function satisfying: (a) $0 \leq \varphi(x) \leq 1$ for any $x \in \mathbb{R}^{N}$; (b) $\varphi(x) \equiv 1$ in $B_{1}$; (c) $\varphi(x) \equiv 0$ in $\mathbb{R}^{N} \backslash \overline{B_{2}}$. Here, $B_{s}$ denotes the ball in $\mathbb{R}^{N}$ of center at origin and radius $s$.

$$
U_{\epsilon}(x)=\frac{\left(N(N-2) \epsilon^{2}\right)^{\frac{N-2}{4}}}{\left(\epsilon^{2}+|x|^{2}\right)^{\frac{N-2}{2}}},
$$

where $U_{1}(x)$ is the extremal function of

$$
S_{\alpha}:=\inf _{u \in D^{1,2}\left(\mathbb{R}^{N}\right) \backslash\{0\}} \frac{\int_{\mathbb{R}^{N}}|\nabla u|^{2}}{\left(\int_{\mathbb{R}^{N}}\left(I_{\alpha} *|u|^{\bar{p}}\right)|u|^{\bar{p}}\right)^{1 / \bar{p}}} .
$$

In [11], they proved that $S_{\alpha}=\frac{S}{\left(A_{\alpha}(N) C_{\alpha}(N)\right)^{1 / \bar{p}}}$, where $A_{\alpha}(N)$ is defined in (1.2), $C_{\alpha}(N)$ is in Lemma 2.2 and

$$
S:=\inf _{u \in D^{1,2}\left(\mathbb{R}^{N}\right) \backslash\{0\}} \frac{\int_{\mathbb{R}^{N}}|\nabla u|^{2}}{\left(\int_{\mathbb{R}^{N}}|u|^{\frac{2 N}{N-2}}\right)^{\frac{N-2}{N}}} .
$$

By [5] (see also [39]), we have the following estimates.

$$
\int_{\mathbb{R}^{N}}\left|\nabla u_{\epsilon}\right|^{2}=S^{\frac{N}{2}}+O\left(\epsilon^{N-2}\right), N \geq 3,
$$

and

$$
\int_{\mathbb{R}^{N}}\left|u_{\epsilon}\right|^{2}= \begin{cases}K_{2} \epsilon^{2}+O\left(\epsilon^{N-2}\right), & N \geq 5 \\ K_{2} \epsilon^{2}|\ln \epsilon|+O\left(\epsilon^{2}\right), & N=4 \\ K_{2} \epsilon+O\left(\epsilon^{2}\right), & N=3\end{cases}
$$


where $K_{2}>0$. By direct calculations, for $t \in\left(2,2^{*}\right)$, there exists $K_{1}>0$ such that

$$
\begin{aligned}
\int_{\mathbb{R}^{N}}\left|u_{\epsilon}\right|^{t} \geq & (N(N-2))^{\frac{N-2}{4} t} \epsilon^{N-\frac{N-2}{2} t} \int_{B_{\frac{1}{\epsilon}}(0)} \frac{1}{\left(1+|x|^{2}\right)^{\frac{N-2}{2} t} d x} \\
& \geq \begin{cases}K_{1} \epsilon^{N-\frac{N-2}{2} t}, & (N-2) t>N, \\
K_{1} \epsilon^{N-\frac{N-2}{2} t}|\ln \epsilon|, & (N-2) t=N, \\
K_{1} \epsilon^{\frac{N-2}{2} t}, & (N-2) t<N .\end{cases}
\end{aligned}
$$

Moreover, similarly as in [10] and [11, by direct computations, we have

$$
\int_{\mathbb{R}^{N}}\left(I_{\alpha} *\left|u_{\epsilon}\right|^{\bar{p}}\right)\left|u_{\epsilon}\right|^{\bar{p}} \geq\left(A_{\alpha}(N) C_{\alpha}(N)\right)^{\frac{N}{2}} S_{\alpha}^{\frac{N+\alpha}{2}}+O\left(\epsilon^{\frac{N+\alpha}{2}}\right) .
$$

Define $v_{\epsilon}(x)=\left(a^{-1}\left\|u_{\epsilon}\right\|_{2}\right)^{\frac{N-2}{2}} u_{\epsilon}\left(a^{-1}\left\|u_{\epsilon}\right\|_{2} x\right)$. Then

$$
\begin{gathered}
\int_{\mathbb{R}^{N}}\left|v_{\epsilon}\right|^{2}=a^{2}, \int_{\mathbb{R}^{N}}\left|\nabla v_{\epsilon}\right|^{2}=\int_{\mathbb{R}^{N}}\left|\nabla u_{\epsilon}\right|^{2}, \\
\int_{\mathbb{R}^{N}}\left(I_{\alpha} *\left|v_{\epsilon}\right|^{\bar{p}}\right)\left|v_{\epsilon}\right|^{\bar{p}}=\int_{\mathbb{R}^{N}}\left(I_{\alpha} *\left|u_{\epsilon}\right|^{\bar{p}}\right)\left|u_{\epsilon}\right|^{\bar{p}},
\end{gathered}
$$

and for $q \in\left[q^{*}, 2^{*}\right)$,

$$
\begin{aligned}
\int_{\mathbb{R}^{N}}\left|v_{\epsilon}\right|^{q} & =\left(a^{-1}\left\|u_{\epsilon}\right\|_{2}\right)^{\frac{N-2}{2} q-N} \int_{\mathbb{R}^{N}}\left|u_{\epsilon}\right|^{q} \\
& \geq a^{N-\frac{N-2}{2} q}\left\|u_{\epsilon}\right\|_{2}^{\frac{N-2}{2} q-N} K_{1} \epsilon^{N-\frac{N-2}{2} q} \\
& \geq \frac{1}{2} a^{N-\frac{N-2}{2} q} K_{1} K_{2}^{\frac{N-2}{4} q-\frac{N}{2}} \times \begin{cases}1, & N \geq 5, \\
|\ln \epsilon|^{\frac{N-2}{4} q-\frac{N}{2}}, & N=4, \\
\epsilon^{\frac{N}{2}-\frac{N-2}{4} q}, & N=3 .\end{cases}
\end{aligned}
$$

Next we use $v_{\epsilon}$ to estimate $c^{p o}$. By Lemma 3.1 there exists a unique $s_{\epsilon}$ such that $P\left(s_{\epsilon} \star v_{\epsilon}\right)=0$ and $E\left(s_{\epsilon} \star v_{\epsilon}\right)=\max _{s \in \mathbb{R}} E\left(s \star v_{\epsilon}\right)$. Thus, $c^{p o} \leq \max _{s \in \mathbb{R}} E\left(s \star v_{\epsilon}\right)$. By direct calculations, one has

$$
\begin{aligned}
& E\left(s \star v_{\epsilon}\right) \\
& =\frac{1}{2} e^{2 s} \int_{\mathbb{R}^{N}}\left|\nabla v_{\epsilon}\right|^{2}-\frac{1}{2 \bar{p}} e^{(N \bar{p}-N-\alpha) s} \int_{\mathbb{R}^{N}}\left(I_{\alpha} *\left|v_{\epsilon}\right|^{\bar{p}}\right)\left|v_{\epsilon}\right|^{\bar{p}}-\frac{\mu}{q} e^{\left(\frac{N}{2} q-N\right) s} \int_{\mathbb{R}^{N}}\left|v_{\epsilon}\right|^{q} \\
& \leq \frac{1}{2} e^{2 s}\left(S^{\frac{N}{2}}+O\left(\epsilon^{N-2}\right)\right)-\frac{1}{2 \bar{p}} e^{(N \bar{p}-N-\alpha) s}\left(\left(A_{\alpha}(N) C_{\alpha}(N)\right)^{\frac{N}{2}} S_{\alpha}^{\frac{N+\alpha}{2}}+O\left(\epsilon^{\frac{N+\alpha}{2}}\right)\right) \\
& \quad-\frac{\mu}{q} e^{\left(\frac{N}{2} q-N\right) s} \frac{1}{2} a^{N-\frac{N-2}{2} q} K_{1} K_{2}^{\frac{N-2}{4} q-\frac{N}{2}} \times \begin{cases}1, & N \geq 5, \\
|\ln \epsilon|^{\frac{N-2}{4} q-\frac{N}{2}}, & N=4, \\
\epsilon^{\frac{N}{2}-\frac{N-2}{4} q}, & N=3 .\end{cases}
\end{aligned}
$$

We claim that there exist $s_{0}, s_{1}>0$ independent of $\epsilon$ such that $s_{\epsilon} \in\left[-s_{0}, s_{1}\right]$ for $\epsilon>0$ small. Suppose by contradiction that $s_{\epsilon} \rightarrow-\infty$ or $s_{\epsilon} \rightarrow+\infty$ as $\epsilon \rightarrow 0$. (3.8) implies that $\max _{s \in \mathbb{R}} E\left(s \star v_{\epsilon}\right) \leq 0$ as $\epsilon \rightarrow 0$ and then $c^{p o} \leq 0$, which contradicts Lemma 3.2 Thus, the claim holds. 
In (3.8), $O\left(\epsilon^{N-2}\right)$ and $O\left(\epsilon^{\frac{N+\alpha}{2}}\right)$ can be controlled by the last term for $\epsilon>0$ small enough. Hence,

$$
\begin{aligned}
\max _{s \in \mathbb{R}} E\left(s \star v_{\epsilon}\right) & <\sup _{s \in \mathbb{R}}\left(\frac{1}{2} e^{2 s} S^{\frac{N}{2}}-\frac{1}{2 \bar{p}} e^{(N \bar{p}-N-\alpha) s}\left(A_{\alpha}(N) C_{\alpha}(N)\right)^{\frac{N}{2}} S_{\alpha}^{\frac{N+\alpha}{2}}\right) \\
& \leq \frac{2+\alpha}{2(N+\alpha)} S_{\alpha}^{\frac{N+\alpha}{2+\alpha}} .
\end{aligned}
$$

The proof is complete.

Lemma 3.5. Assume the conditions in Theorem 1.2 hold. If $u \in \mathcal{P}$ such that $E(u)=c^{p o}$, then $u$ satisfies the equation (1.1) with some $\lambda<0$.

Proof. By the Lagrange multipliers rule, there exist $\lambda$ and $\eta$ such that $u$ satisfies

$$
\begin{aligned}
& -\Delta u-\left(I_{\alpha} *|u|^{p}\right)|u|^{p-2} u-\mu|u|^{q-2} u \\
& \quad=\lambda u+\eta\left[-2 \Delta u-2 p \eta_{p}\left(I_{\alpha} *|u|^{p}\right)|u|^{p-2} u-\mu q \gamma_{q}|u|^{q-2} u\right],
\end{aligned}
$$

or equivalently,

$$
-(1-2 \eta) \Delta u=\lambda u+\left(1-\eta 2 p \eta_{p}\right)\left(I_{\alpha} *|u|^{p}\right)|u|^{p-2} u+\mu\left(1-\eta q \gamma_{q}\right)|u|^{q-2} u .
$$

Next we show $\eta=0$. Similarly to the definition of $P(u)$ (see Lemma 2.8), we obtain

$$
(1-2 \eta) \int_{\mathbb{R}^{N}}|\nabla u|^{2}-\left(1-\eta 2 p \eta_{p}\right) \eta_{p} \int_{\mathbb{R}^{N}}\left(I_{\alpha} *|u|^{p}\right)|u|^{p}-\mu\left(1-\eta q \gamma_{q}\right) \gamma_{q} \int_{\mathbb{R}^{N}}|u|^{q}=0
$$

which combined with $P(u)=0$ gives that

$$
\eta\left(2 \int_{\mathbb{R}^{N}}|\nabla u|^{2}-2 p \eta_{p}^{2} \int_{\mathbb{R}^{N}}\left(I_{\alpha} *|u|^{p}\right)|u|^{p}-\mu q \gamma_{q}^{2} \int_{\mathbb{R}^{N}}|u|^{q}\right)=0 .
$$

If $\eta \neq 0$, then

$$
2 \int_{\mathbb{R}^{N}}|\nabla u|^{2}-2 p \eta_{p}^{2} \int_{\mathbb{R}^{N}}\left(I_{\alpha} *|u|^{p}\right)|u|^{p}-\mu q \gamma_{q}^{2} \int_{\mathbb{R}^{N}}|u|^{q}=0,
$$

which combined with $P(u)=0$ gives that

$$
\left\{\begin{array}{l}
\mu \gamma_{q}\left(2 p \eta_{p}-q \gamma_{q}\right) \int_{\mathbb{R}^{N}}|u|^{q}=\left(2 p \eta_{p}-2\right) \int_{\mathbb{R}^{N}}|\nabla u|^{2} \\
\eta_{p}\left(q \gamma_{q}-2 p \eta_{p}\right) \int_{\mathbb{R}^{N}}\left(I_{\alpha} *|u|^{p}\right)|u|^{p}=\left(q \gamma_{q}-2\right) \int_{\mathbb{R}^{N}}|\nabla u|^{2} .
\end{array}\right.
$$

That is a contradiction. So $\eta=0$.

From (3.9) with $\eta=0, P(u)=0,0<\gamma_{q}<1,0<\eta_{p} \leq 1$ and $\mu>0$, we obtain

$$
\begin{aligned}
\lambda a^{2} & =\int_{\mathbb{R}^{N}}|\nabla u|^{2}-\int_{\mathbb{R}^{N}}\left(I_{\alpha} *|u|^{p}\right)|u|^{p}-\mu \int_{\mathbb{R}^{N}}|u|^{q} \\
& =\left(\eta_{p}-1\right) \int_{\mathbb{R}^{N}}\left(I_{\alpha} *|u|^{p}\right)|u|^{p}+\mu\left(\gamma_{q}-1\right) \int_{\mathbb{R}^{N}}|u|^{q}<0,
\end{aligned}
$$

which implies $\lambda<0$. The proof is complete.

Lemma 3.6. Assume the conditions in Theorem 1.2 hold. If $\mathcal{C} \neq \emptyset$, then $c^{p o}=c^{g}$ and $\mathcal{C}=\mathcal{G}$, where $\mathcal{C}:=\left\{u \in \mathcal{P}: E(u)=c^{\text {po }}\right\}$.

Proof. For any $u \in \mathcal{C}$, by Lemma 3.5 $u$ is a solution to (1.1) and thus $E(u) \geq c^{g}$. So $c^{p o} \geq c^{g}$ holds. On the other hand, for any normalized solution $v$ of (1.1) on $S_{a}$, by Lemma 2.8, $P(v)=0$ and thus $E(v) \geq c^{p o}$, which implies that the reverse inequality $c^{g} \geq c^{p o}$ holds. Hence $c^{g}=c^{p o}$ and $\mathcal{C}=\mathcal{G}$. 
Proposition 3.7. Assume the conditions in Theorem 1.2 hold. Let $\left\{u_{n}\right\} \subset S_{a, r}$ be a Palais-Smale sequence for $\left.E\right|_{s_{a}}$ at level $c^{\text {po }}$ with $P\left(u_{n}\right) \rightarrow 0$ as $n \rightarrow \infty$. Then up to a subsequence, $u_{n} \rightarrow u$ strongly in $H^{1}\left(\mathbb{R}^{N}\right)$, and $u \in S_{a}$ is a radial solution to (1.1) with some $\lambda<0$.

Proof. The proof is divided into four steps.

Step 1. We show $\left\{u_{n}\right\}$ is bounded in $H^{1}\left(\mathbb{R}^{N}\right)$. Since $\left\{u_{n}\right\} \subset S_{a}$, it is enough to show that $\left\{\left\|\nabla u_{n}\right\|_{2}^{2}\right\}$ is bounded.

Case $q>q^{*}$, it follows from $P\left(u_{n}\right)=o_{n}(1)$ and $E\left(u_{n}\right)=c^{p o}+o_{n}(1)$ that

$$
E\left(u_{n}\right)=\left(\frac{\eta_{p}}{2}-\frac{1}{2 p}\right) \int_{\mathbb{R}^{N}}\left(I_{\alpha} *\left|u_{n}\right|^{p}\right)\left|u_{n}\right|^{p}+\left(\frac{\gamma_{q}}{2}-\frac{1}{q}\right) \mu \int_{\mathbb{R}^{N}}\left|u_{n}\right|^{q}+o_{n}(1),
$$

and then

$$
\left(\frac{\eta_{p}}{2}-\frac{1}{2 p}\right) \int_{\mathbb{R}^{N}}\left(I_{\alpha} *\left|u_{n}\right|^{p}\right)\left|u_{n}\right|^{p}+\left(\frac{\gamma_{q}}{2}-\frac{1}{q}\right) \mu \int_{\mathbb{R}^{N}}\left|u_{n}\right|^{q} \leq C .
$$

Using $P\left(u_{n}\right)=o_{n}(1)$ again yields that

$$
\int_{\mathbb{R}^{N}}\left|\nabla u_{n}\right|^{2}=\eta_{p} \int_{\mathbb{R}^{N}}\left(I_{\alpha} *\left|u_{n}\right|^{p}\right)\left|u_{n}\right|^{p}+\mu \gamma_{q} \int_{\mathbb{R}^{N}}\left|u_{n}\right|^{q}+o_{n}(1) \leq C .
$$

Case $q=q^{*}$, from the equality

$$
E\left(u_{n}\right)=\left(\frac{\eta_{p}}{2}-\frac{1}{2 p}\right) \int_{\mathbb{R}^{N}}\left(I_{\alpha} *\left|u_{n}\right|^{p}\right)\left|u_{n}\right|^{p}+o_{n}(1),
$$

we know that $\left\{\int_{\mathbb{R}^{N}}\left(I_{\alpha} *\left|u_{n}\right|^{p}\right)\left|u_{n}\right|^{p}\right\}$ is bounded. Suppose by contradiction that $\left\{\left\|\nabla u_{n}\right\|_{2}^{2}\right\}$ is not bounded, then by $P\left(u_{n}\right)=o_{n}(1)$, we obtain that

$$
\lim _{n \rightarrow \infty} \frac{\mu \gamma_{q} \int_{\mathbb{R}^{N}}\left|u_{n}\right|^{q}}{\int_{\mathbb{R}^{N}}\left|\nabla u_{n}\right|^{2}}=1
$$

which contradicts the fact

$$
\mu \gamma_{q} \int_{\mathbb{R}^{N}}\left|u_{n}\right|^{q} \leq \mu \gamma_{q} C_{N, q}^{q} a^{q\left(1-\gamma_{q}\right)}\left\|\nabla u_{n}\right\|_{2}^{2}<\left\|\nabla u_{n}\right\|_{2}^{2} .
$$

Hence, $\left\{\left\|\nabla u_{n}\right\|_{2}^{2}\right\}$ is bounded.

There exists $u \in H_{r}^{1}\left(\mathbb{R}^{N}\right)$ such that, up to a subsequence, $u_{n} \rightarrow u$ weakly in $H^{1}\left(\mathbb{R}^{N}\right), u_{n} \rightarrow u$ strongly in $L^{t}\left(\mathbb{R}^{N}\right)$ with $t \in\left(2,2^{*}\right)$ and $u_{n} \rightarrow u$ a.e. in $\mathbb{R}^{N}$.

Step 2. We claim that $u \neq \equiv 0$. Suppose by contradiction that $u \equiv 0$.

Case $p<\bar{p}$. By the assumptions on $p$ and $q$, we have

$$
\begin{aligned}
E\left(u_{n}\right) & =\left(\frac{\eta_{p}}{2}-\frac{1}{2 p}\right) \int_{\mathbb{R}^{N}}\left(I_{\alpha} *\left|u_{n}\right|^{p}\right)\left|u_{n}\right|^{p}+\left(\frac{\gamma_{q}}{2}-\frac{1}{q}\right) \mu \int_{\mathbb{R}^{N}}\left|u_{n}\right|^{q}+o_{n}(1) \\
& =o_{n}(1),
\end{aligned}
$$

which contradicts $E\left(u_{n}\right) \rightarrow c^{p o}>0$.

Case $p=\bar{p}$. By using $E\left(u_{n}\right)=c^{p o}+o_{n}(1), P\left(u_{n}\right)=o_{n}(1), \int_{\mathbb{R}^{N}}\left|u_{n}\right|^{q}=o_{n}(1)$ and (3.5), we get that

$$
E\left(u_{n}\right)=\left(\frac{1}{2}-\frac{1}{2 p \eta_{p}}\right) \int_{\mathbb{R}^{N}}\left|\nabla u_{n}\right|^{2}+o_{n}(1)
$$


and

$$
\begin{aligned}
\int_{\mathbb{R}^{N}}\left|\nabla u_{n}\right|^{2} & =\eta_{p} \int_{\mathbb{R}^{N}}\left(I_{\alpha} *\left|u_{n}\right|^{p}\right)\left|u_{n}\right|^{p}+o_{n}(1) \\
& \leq \eta_{p}\left(\frac{\int_{\mathbb{R}^{N}}\left|\nabla u_{n}\right|^{2}}{S_{\alpha}}\right)^{p}+o_{n}(1) .
\end{aligned}
$$

Since $c^{p o}>0$, we obtain $\lim \inf _{n \rightarrow \infty} \int_{\mathbb{R}^{N}}\left|\nabla u_{n}\right|^{2}>0$ and hence

$$
\limsup _{n \rightarrow \infty}\left\|\nabla u_{n}\right\|_{2}^{2} \geq S_{\alpha}^{\frac{N+\alpha}{2+\alpha}} \text {. }
$$

Consequently,

$$
c^{p o}=\lim _{n \rightarrow \infty}\left\{\left(\frac{1}{2}-\frac{1}{2 p \eta_{p}}\right) \int_{\mathbb{R}^{N}}\left|\nabla u_{n}\right|^{2}+o_{n}(1)\right\} \geq \frac{2+\alpha}{2(N+\alpha)} S_{\alpha}^{\frac{N+\alpha}{2+\alpha}},
$$

which contradicts Lemma 3.4. So $u \not \equiv 0$.

Step 3. We show $u$ is a solution to (1.1) with some $\lambda<0$. Since $\left\{u_{n}\right\}$ is a Palais-Smale sequence of $\left.E\right|_{S_{a}}$, by the Lagrange multipliers rule, there exists $\lambda_{n}$ such that

$$
\int_{\mathbb{R}^{N}}\left(\nabla u_{n} \cdot \nabla \varphi-\lambda_{n} u_{n} \varphi-\left(I_{\alpha} *\left|u_{n}\right|^{p}\right)\left|u_{n}\right|^{p-2} u_{n} \varphi-\mu\left|u_{n}\right|^{q-2} u_{n} \varphi\right)=o_{n}(1)\|\varphi\|
$$

for every $\varphi \in H^{1}\left(\mathbb{R}^{N}\right)$. The choice $\varphi=u_{n}$ provides

$$
\lambda_{n} a^{2}=\int_{\mathbb{R}^{N}}\left|\nabla u_{n}\right|^{2}-\int_{\mathbb{R}^{N}}\left(I_{\alpha} *\left|u_{n}\right|^{p}\right)\left|u_{n}\right|^{p}-\mu \int_{\mathbb{R}^{N}}\left|u_{n}\right|^{q}+o_{n}(1)
$$

and the boundedness of $\left\{u_{n}\right\}$ in $H^{1}\left(\mathbb{R}^{N}\right)$ implies that $\lambda_{n}$ is bounded as well; thus, up to a subsequence $\lambda_{n} \rightarrow \lambda \in \mathbb{R}^{N}$. Furthermore, by using $P\left(u_{n}\right)=o_{n}(1)$, (3.13), $\mu>0, \eta_{p} \in(0,1], \gamma_{q} \in(0,1)$ and $u_{n} \rightarrow u$ weakly in $H^{1}\left(\mathbb{R}^{N}\right)$, we have

$$
-\lambda_{n} a^{2}=\left(1-\eta_{p}\right) \int_{\mathbb{R}^{N}}\left(I_{\alpha} *\left|u_{n}\right|^{p}\right)\left|u_{n}\right|^{p}+\mu\left(1-\gamma_{q}\right) \int_{\mathbb{R}^{N}}\left|u_{n}\right|^{q}+o_{n}(1)
$$

and then

$$
-\lambda a^{2} \geq\left(1-\eta_{p}\right) \int_{\mathbb{R}^{N}}\left(I_{\alpha} *|u|^{p}\right)|u|^{p}+\mu\left(1-\gamma_{q}\right) \int_{\mathbb{R}^{N}}|u|^{q}>0,
$$

which implies that $\lambda<0$. By using (3.12) and Lemma 2.6, we obtain that

$$
\begin{aligned}
& \int_{\mathbb{R}^{N}}\left(\nabla u \cdot \nabla \varphi-\lambda u \varphi-\left(I_{\alpha} *|u|^{p}\right)|u|^{p-2} u \varphi-\mu|u|^{q-2} u \varphi\right) \\
& =\lim _{n \rightarrow \infty} \int_{\mathbb{R}^{N}}\left(\nabla u_{n} \cdot \nabla \varphi-\lambda_{n} u_{n} \varphi-\left(I_{\alpha} *\left|u_{n}\right|^{p}\right)\left|u_{n}\right|^{p-2} u_{n} \varphi-\mu\left|u_{n}\right|^{q-2} u_{n} \varphi\right) \\
& =\lim _{n \rightarrow \infty} o_{n}(1)\|\varphi\|=0,
\end{aligned}
$$

which implies that $u$ satisfies the equation

$$
-\Delta u=\lambda u+\left(I_{\alpha} *|u|^{p}\right)|u|^{p-2} u+\mu|u|^{q-2} u .
$$

Thus, $P(u)=0$ by Lemma 2.8 .

Step 4. We show $u_{n} \rightarrow u$ strongly in $H^{1}\left(\mathbb{R}^{N}\right)$. 
Case $p<\bar{p}$. Choosing $\varphi=u_{n}-u$ in (3.12) and (3.14), and subtracting, we obtain that

$$
\int_{\mathbb{R}^{N}}\left(\left|\nabla\left(u_{n}-u\right)\right|^{2}-\lambda\left|u_{n}-u\right|^{2}\right) \rightarrow 0 .
$$

Since $\lambda<0$, we have $u_{n} \rightarrow u$ strongly in $H^{1}\left(\mathbb{R}^{N}\right)$.

Case $p=\bar{p}$. Set $v_{n}:=u_{n}-u$. Then we have

$$
\begin{gathered}
\left\|u_{n}\right\|_{2}^{2}=\|u\|_{2}^{2}+\left\|v_{n}\right\|_{2}^{2}+o_{n}(1),\left\|\nabla u_{n}\right\|_{2}^{2}=\|\nabla u\|_{2}^{2}+\left\|\nabla v_{n}\right\|_{2}^{2}+o_{n}(1), \\
\left\|u_{n}\right\|_{q}^{q}=\|u\|_{q}^{q}+\left\|v_{n}\right\|_{q}^{q}+o_{n}(1)=\|u\|_{q}^{q}+o_{n}(1)
\end{gathered}
$$

and

$$
\int_{\mathbb{R}^{N}}\left(I_{\alpha} *\left|u_{n}\right|^{p}\right)\left|u_{n}\right|^{p}=\int_{\mathbb{R}^{N}}\left(I_{\alpha} *|u|^{p}\right)|u|^{p}+\int_{\mathbb{R}^{N}}\left(I_{\alpha} *\left|v_{n}\right|^{p}\right)\left|v_{n}\right|^{p}+o_{n}(1),
$$

which combined with $P\left(u_{n}\right)=o_{n}(1)$ and $P(u)=0$ gives that

$$
\int_{\mathbb{R}^{N}}\left|\nabla v_{n}\right|^{2}=\eta_{p} \int_{\mathbb{R}^{N}}\left(I_{\alpha} *\left|v_{n}\right|^{p}\right)\left|v_{n}\right|^{p}+o_{n}(1) .
$$

Similarly to (3.11), we infer that

$$
\limsup _{n \rightarrow \infty}\left\|\nabla v_{n}\right\|_{2}^{2} \geq S_{\alpha}^{\frac{N+\alpha}{2+\alpha}} \text { or } \liminf _{n \rightarrow \infty}\left\|\nabla v_{n}\right\|_{2}^{2}=0 .
$$

If $\lim \sup _{n \rightarrow \infty}\left\|\nabla v_{n}\right\|_{2}^{2} \geq S_{\alpha}^{\frac{N+\alpha}{2+\alpha}}$, then by $2 p \eta_{p} \geq 2, q \gamma_{q} \geq 2, \mu>0$ and (3.16),

$$
\begin{aligned}
E\left(u_{n}\right) & =\left(\frac{\eta_{p}}{2}-\frac{1}{2 p}\right) \int_{\mathbb{R}^{N}}\left(I_{\alpha} *\left|u_{n}\right|^{p}\right)\left|u_{n}\right|^{p}+\left(\frac{\gamma_{q}}{2}-\frac{1}{q}\right) \mu \int_{\mathbb{R}^{N}}\left|u_{n}\right|^{q}+o_{n}(1) \\
& \geq\left(\frac{\eta_{p}}{2}-\frac{1}{2 p}\right) \int_{\mathbb{R}^{N}}\left(I_{\alpha} *\left|v_{n}\right|^{p}\right)\left|v_{n}\right|^{p}+o_{n}(1) \\
& \geq \frac{2+\alpha}{2(N+\alpha)} S_{\alpha}^{\frac{N+\alpha}{2+\alpha}}+o_{n}(1),
\end{aligned}
$$

which contradicts $E\left(u_{n}\right)=c^{p o}+o_{n}(1)$ and Lemma 3.4. Thus $\lim \inf _{n \rightarrow \infty}\left\|\nabla v_{n}\right\|_{2}^{2}=$ 0 holds. So up to a subsequence, $\nabla u_{n} \rightarrow \nabla u$ in $L^{2}\left(\mathbb{R}^{N}\right)$. Hence, similarly to the case $p<\bar{p}$, we infer that $u_{n} \rightarrow u$ strongly in $H^{1}\left(\mathbb{R}^{N}\right)$. The proof is complete.

Now we are ready to give the proof of Theorem 1.2

Proof of Theorem 1.2. Let $k>0$ be defined by Lemma 3.3. Following the strategy in [17] and [34] (see also [15]), we consider the augmented functional $\tilde{E}$ : $\mathbb{R} \times H^{1}\left(\mathbb{R}^{N}\right) \rightarrow \mathbb{R}$ defined by

$$
\begin{gathered}
\tilde{E}(s, u):=E(s \star u)=\frac{1}{2} e^{2 s} \int_{\mathbb{R}^{N}}|\nabla u|^{2}-\frac{1}{2 p} e^{(N p-N-\alpha) s} \int_{\mathbb{R}^{N}}\left(I_{\alpha} *|u|^{p}\right)|u|^{p} \\
-\frac{\mu}{q} e^{\left(\frac{N}{2} q-N\right) s} \int_{\mathbb{R}^{N}}|u|^{q},
\end{gathered}
$$

and consider the restriction $\left.\tilde{E}\right|_{\mathbb{R} \times S_{a, r}}$. Note that $\tilde{E} \in C^{1}$. Denoting by $E^{c}$ the closed sub-level set $\left\{u \in S_{a}: E(u) \leq c\right\}$, we introduce the mini-max class

$$
\Gamma_{r}:=\left\{\gamma=(\kappa, \beta) \in C\left([0,1], \mathbb{R} \times S_{a, r}\right): \gamma(0) \in\left(0, \overline{A_{k}}\right), \gamma(1) \in\left(0, E^{0}\right)\right\}
$$

with associated mini-max level

$$
c_{r}^{m p}:=\inf _{\gamma \in \Gamma_{r}} \max _{(s, u) \in \gamma([0,1])} \tilde{E}(s, u) .
$$


Let $u \in S_{a, r}$. Since $\int_{\mathbb{R}^{N}}|\nabla(s \star u)|^{2} \rightarrow 0^{+}$as $s \rightarrow-\infty$ and $E(s \star u) \rightarrow-\infty$ as $s \rightarrow+\infty$, there exist $s_{0} \ll 1$ and $s_{1} \gg 1$ such that

$$
\gamma_{u}: \tau \in[0,1] \mapsto\left(0,\left((1-\tau) s_{0}+\tau s_{1}\right) \star u\right) \in \mathbb{R} \times S_{a, r}
$$

is a path in $\Gamma_{r}$. The continuity of $\gamma_{u}$ follows from the fact that

$$
(s, u) \in \mathbb{R} \times H^{1}\left(\mathbb{R}^{N}\right) \mapsto(s \star u) \in H^{1}\left(\mathbb{R}^{N}\right) \text { is continuous, }
$$

see Lemma 3.5 in [2]. Hence $c_{r}^{m p}$ is well defined.

To study the value of $c_{r}^{m p}$, we also consider the mini-max level

$$
c^{m p}:=\inf _{\gamma \in \Gamma} \max _{(s, u) \in \gamma([0,1])} \tilde{E}(s, u)
$$

with

$$
\Gamma:=\left\{\gamma=(\kappa, \beta) \in C\left([0,1], \mathbb{R} \times S_{a}\right): \gamma(0) \in\left(0, \overline{A_{k}}\right), \gamma(1) \in\left(0, E^{0}\right)\right\} .
$$

Obviously, $c_{r}^{m p} \geq c^{m p}$.

For any $\gamma=(\kappa, \beta) \in \Gamma$, consider the function

$$
P_{\gamma}: \tau \in[0,1] \mapsto P(\kappa(\tau) \star \beta(\tau)) \in \mathbb{R} .
$$

We have $P_{\gamma}(0)=P(\beta(0))>0$ by Lemma 3.3, and by Lemma 3.1, $P_{\gamma}(1)=$ $P(\beta(1))<0$ since $E(\beta(1)) \leq 0$. Moreover, $P_{\gamma}$ is continuous by (3.20), and hence there exists $\tau_{\gamma} \in(0,1)$ such that $P_{\gamma}\left(\tau_{\gamma}\right)=0$, namely $\kappa\left(\tau_{\gamma}\right) \star \beta\left(\tau_{\gamma}\right) \in \mathcal{P}$; this implies that

$$
\max _{\gamma([0,1])} \tilde{E} \geq \tilde{E}\left(\gamma\left(\tau_{\gamma}\right)\right)=E\left(\kappa\left(\tau_{\gamma}\right) \star \beta\left(\tau_{\gamma}\right)\right) \geq \inf _{\mathcal{P}} E=c^{p o},
$$

and consequently $c^{m p} \geq c^{p o}$.

For any $u \in \mathcal{P}$, let $|u|^{*}$ be the Schwartz symmetrization rearrangement of $|u|$. Since $\left\||u|^{*}\right\|_{t}=\|u\|_{t}$ with $t \in[1, \infty),\left\|\nabla\left(|u|^{*}\right)\right\|_{2} \leq\|\nabla u\|_{2}$ and

$$
\int_{\mathbb{R}^{N}}\left(I_{\alpha} *\left(|u|^{*}\right)^{p}\right)\left(|u|^{*}\right)^{p} \geq \int_{\mathbb{R}^{N}}\left(I_{\alpha} *|u|^{p}\right)|u|^{p},
$$

we obtain that $\Psi_{|u|^{*}}(s) \leq \Psi_{u}(s)$ for any $s \in \mathbb{R}$, where $\Psi_{u}(s)$ is defined in (3.2). Let $s_{u}$ be defined by Lemma 3.1 be such that $P\left(s_{u} \star u\right)=0$. Then

$$
E(u)=\Psi_{u}(0)=\Psi_{u}\left(s_{u}\right) \geq \Psi_{u}\left(s_{|u|^{*}}\right) \geq \Psi_{|u|^{*}}\left(s_{|u|^{*}}\right) .
$$

Since $s_{|u|^{*}} \star|u|^{*} \in \mathcal{P} \cap H_{r}^{1}\left(\mathbb{R}^{N}\right)$, we have that

$$
E(u) \geq \inf _{\mathcal{P} \cap H_{r}^{1}\left(\mathbb{R}^{N}\right)} E(u),
$$

which implies that

$$
c^{p o}=\inf _{\mathcal{P}} E(u) \geq \inf _{\mathcal{P} \cap H_{r}^{1}\left(\mathbb{R}^{N}\right)} E(u) .
$$

For any $u \in \mathcal{P} \cap H_{r}^{1}\left(\mathbb{R}^{N}\right), \gamma_{u}$ defined in (3.19) is a path in $\Gamma_{r}$ with

$$
E(u)=\max _{\gamma_{u}([0,1])} \tilde{E} \geq c_{r}^{m p}
$$

which implies

$$
\inf _{\mathcal{P} \cap H_{r}^{1}\left(\mathbb{R}^{N}\right)} E(u) \geq c_{r}^{m p} .
$$

Now, we have proved that

$$
c^{p o}=c^{m p}=c_{r}^{m p}>\sup _{\left(\overline{A_{k}} \cup E^{0}\right) \cap S_{a, r}} E=\sup _{\left(\left(0, \overline{A_{k}}\right) \cup\left(0, E^{0}\right)\right) \cap\left(\mathbb{R} \times S_{a, r}\right)} \tilde{E} .
$$


Using the terminology of Section 5 in [12, this means that $\left\{\gamma([0,1]): \gamma \in \Gamma_{r}\right\}$ is a homotopy stable family of compact subsets of $\mathbb{R} \times S_{a, r}$ with extended closed boundary $\left(0, \overline{A_{k}}\right) \cup\left(0, E^{0}\right)$, and that the super-level set $\left\{\tilde{E} \geq c^{p o}\right\}$ is a dual set for $\Gamma_{r}$, in the sense that assumptions $\left(F^{\prime} 1\right)$ and $\left(F^{\prime} 2\right)$ in Theorem 5.2 of [12] are satisfied. Therefore, taking any minimizing sequence $\left\{\gamma_{n}=\left(\kappa_{n}, \beta_{n}\right)\right\} \subset \Gamma_{r}$ for $c^{p o}$ with the property that $\kappa_{n} \equiv 0$ and $\beta_{n}(\tau) \geq 0$ a.e. in $\mathbb{R}^{N}$ for every $\tau \in[0,1]$, there exists a Palais-Smale sequence $\left\{\left(s_{n}, w_{n}\right)\right\} \subset \mathbb{R} \times S_{a, r}$ for $\left.\tilde{E}\right|_{\mathbb{R} \times S_{a, r}}$ at level $c^{p o}$, that is, $\tilde{E}\left(s_{n}, w_{n}\right) \rightarrow c^{p o}$,

$$
\partial_{s} \tilde{E}\left(s_{n}, w_{n}\right) \rightarrow 0 \text { and }\left\|\partial_{u} \tilde{E}\left(s_{n}, w_{n}\right)\right\|_{\left(T_{w_{n}} S_{a, r}\right)^{*}} \rightarrow 0 \text { as } n \rightarrow \infty
$$

with the additional property that

$$
\left|s_{n}\right|+\operatorname{dist}_{H^{1}\left(\mathbb{R}^{N}\right)}\left(w_{n}, \beta_{n}([0,1])\right) \rightarrow 0 \text { as } n \rightarrow \infty .
$$

By (3.26), the first condition in (3.26) reads $P\left(s_{n} \star w_{n}\right) \rightarrow 0$, while the second condition gives that

$$
\begin{array}{r}
e^{2 s_{n}} \int_{\mathbb{R}^{N}} \nabla w_{n} \cdot \nabla \phi-e^{(N p-N-\alpha) s_{n}} \int_{\mathbb{R}^{N}}\left(I_{\alpha} *\left|w_{n}\right|^{p}\right)\left|w_{n}\right|^{p-2} w_{n} \phi \\
-\mu e^{\left(\frac{N}{2} q-N\right) s_{n}} \int_{\mathbb{R}^{N}}\left|w_{n}\right|^{q-2} w_{n} \phi=o_{n}(1)\|\phi\|
\end{array}
$$

for every $\phi \in T_{w_{n}} S_{a, r}$. Since $\left\{s_{n}\right\}$ is bounded due to (3.27), this is equivalent to

$$
d E\left(s_{n} \star w_{n}\right)\left[s_{n} \star \phi\right]=o_{n}(1)\|\phi\|=o_{n}(1)\left\|s_{n} \star \phi\right\| \text { as } n \rightarrow \infty .
$$

Let $u_{n}:=s_{n} \star w_{n}$. By Lemma 5.8 in [34, equation (3.28) establishes that $\left\{u_{n}\right\} \subset$ $S_{a, r}$ is a Palais-Smale sequence for $\left.E\right|_{S_{a, r}}$ (thus a Palais-Smale sequence for $\left.E\right|_{S_{a}}$, since the problem is invariant under rotations) at level $c^{p o}$, with $P\left(u_{n}\right) \rightarrow 0$ as $n \rightarrow \infty$.

By Proposition 3.7 up to a subsequence, $u_{n} \rightarrow u$ strongly in $H^{1}\left(\mathbb{R}^{N}\right)$. Thus, $u \in S_{a}$ is a mountain pass type normalized solution to (1.1) with $\lambda<0$ and $E(u)=c^{p o}$. By Lemma 3.6, $u$ is a normalized ground state. The proof is complete.

\section{Proof of Theorem 1.4}

Firstly, we study the positivity of the normalized ground states to (1.1). By Lemma 3.6 it is enough to prove the following fact.

Proposition 4.1. Assume the conditions in Theorem 1.2 hold. If $u \in \mathcal{P}$ such that $E(u)=c^{p o}$, then $|u| \in \mathcal{P}$ and $E(|u|)=c^{p o}$. Moreover, $|u|>0$ in $\mathbb{R}^{N}$.

Proof. It follows from

$$
\int_{\mathbb{R}^{N}}|\nabla| u||^{2} \leq \int_{\mathbb{R}^{N}}|\nabla u|^{2}
$$


that $P(|u|) \leq 0$. By Lemma 3.1 there exists $s_{|u|} \leq 0$ such that $s_{|u|} \star|u| \in \mathcal{P}$. Thus,

$$
\begin{aligned}
& E\left(s_{|u|} \star|u|\right) \\
& =\left(\frac{\eta_{p}}{2}-\frac{1}{2 p}\right) \int_{\mathbb{R}^{N}}\left(I_{\alpha} *\left|s_{|u|} \star\right| u||^{p}\right)\left|s_{|u|} \star\right| u||^{p}+\left.\left(\frac{\gamma_{q}}{2}-\frac{1}{q}\right) \mu \int_{\mathbb{R}^{N}}\left|s_{|u|} \star\right| u\right|^{q} \\
& =\left(\frac{\eta_{p}}{2}-\frac{1}{2 p}\right) e^{(N p-N-\alpha) s_{|u|}} \int_{\mathbb{R}^{N}}\left(I_{\alpha} *|u|^{p}\right)|u|^{p}+\left(\frac{\gamma_{q}}{2}-\frac{1}{q}\right) \mu e^{\left(\frac{N}{2} q-N\right) s_{|u|}} \int_{\mathbb{R}^{N}}|u|^{q} \\
& \leq\left(\frac{\eta_{p}}{2}-\frac{1}{2 p}\right) \int_{\mathbb{R}^{N}}\left(I_{\alpha} *|u|^{p}\right)|u|^{p}+\left(\frac{\gamma_{q}}{2}-\frac{1}{q}\right) \mu \int_{\mathbb{R}^{N}}|u|^{q} \\
& =E(u)=c^{p o} .
\end{aligned}
$$

By the definition of $c^{p o}$, we have $s_{|u|}=0,|u| \in \mathcal{P}$ and $E(|u|)=c^{p o}$.

By Lemma 3.5 there exists $\lambda<0$ such that $|u|$ satisfies the equation

$$
-\Delta u=\lambda u+\left(I_{\alpha} *|u|^{p}\right)|u|^{p-2} u+\mu|u|^{q-2} u .
$$

Since $|u|$ is continuous by Theorem 2.1 in [19], the strong maximum principle implies that $|u|>0$ in $\mathbb{R}^{N}$.

Nextly, we study the radial symmetry of the normalized ground states to (1.1). We follow the strategy of [28]. The argument relies on polarizations. So we first recall some theories of polarizations $([6,27,36])$.

Assume that $H \subset \mathbb{R}^{N}$ is a closed half-space and that $\sigma_{H}$ is the reflection with respect to $\partial H$. The polarization $u^{H}: \mathbb{R}^{N} \rightarrow \mathbb{R}$ of $u: \mathbb{R}^{N} \rightarrow \mathbb{R}$ is defined for $x \in \mathbb{R}^{N}$ by

$$
u^{H}(x)= \begin{cases}\max \left\{u(x), u\left(\sigma_{H}(x)\right)\right\}, & \text { if } x \in H \\ \min \left\{u(x), u\left(\sigma_{H}(x)\right)\right\}, & \text { if } x \notin H .\end{cases}
$$

We will use the following standard property of polarizations (Lemma 5.3 in [6]).

Lemma 4.2. (Polarization and Dirichlet integrals). If $u \in H^{1}\left(\mathbb{R}^{N}\right)$, then $u^{H} \in$ $H^{1}\left(\mathbb{R}^{N}\right)$ and

$$
\int_{\mathbb{R}^{N}}\left|\nabla u^{H}\right|^{2}=\int_{\mathbb{R}^{N}}|\nabla u|^{2}
$$

We shall also use a polarization inequality with equality cases (Lemma 5.3 in [27]).

Lemma 4.3. (Polarization and nonlocal integrals). Let $\alpha \in(0, N), u \in L^{\frac{2 N}{N+\alpha}}\left(\mathbb{R}^{N}\right)$ and $H \subset \mathbb{R}^{N}$ be a closed half-space. If $u \geq 0$, then

$$
\int_{\mathbb{R}^{N}} \int_{\mathbb{R}^{N}} \frac{u(x) u(y)}{|x-y|^{N-\alpha}} d x d y \leq \int_{\mathbb{R}^{N}} \int_{\mathbb{R}^{N}} \frac{u^{H}(x) u^{H}(y)}{|x-y|^{N-\alpha}} d x d y,
$$

with equality if and only if either $u^{H}=u$ or $u^{H}=u \circ \sigma_{H}$.

The last tool that we need is a characterization of symmetric functions by polarizations (Proposition 3.15 in [36], Lemma 5.4 in [27]).

Lemma 4.4. (Symmetry and polarization). Assume that $u \in L^{2}\left(\mathbb{R}^{N}\right)$ is nonnegative. There exist $x_{0} \in \mathbb{R}^{N}$ and a non-increasing function $v:(0, \infty) \rightarrow \mathbb{R}$ such that for almost every $x \in \mathbb{R}^{N}, u(x)=v\left(\left|x-x_{0}\right|\right)$ if and only if for every closed half-space $H \subset \mathbb{R}^{N}, u^{H}=u$ or $u^{H}=u \circ \sigma_{H}$.

Now we are ready to prove the radial symmetry of the positive normalized ground states to (1.1). 
Proposition 4.5. Assume that the conditions in Theorem 1.2 hold. Let $u$ be a positive normalized ground state to (1.1), then there exist $x_{0} \in \mathbb{R}^{N}$ and a nonincreasing positive function $v:(0, \infty) \rightarrow \mathbb{R}$ such that $u(x)=v\left(\left|x-x_{0}\right|\right)$ for almost every $x \in \mathbb{R}^{N}$.

Proof. By Lemma 3.6. $E(u)=c^{p o}$ and $P(u)=0$. Let $\tilde{E}$ and $\Gamma$ be defined in (3.17) and (3.22), respectively, and let $\gamma_{u}(\tau)=\left(0,\left((1-\tau) s_{0}+\tau s_{1}\right) \star u\right) \in \Gamma$ be a path defined in (3.19). Denote $\beta_{u}(\tau)=\left((1-\tau) s_{0}+\tau s_{1}\right) \star u$. Then, $\beta_{u}\left(-s_{0} /\left(s_{1}-s_{0}\right)\right)=$ $u, \beta_{u}(\tau) \geq 0$ for every $\tau \in[0,1], \tilde{E}\left(\gamma_{u}(\tau)\right)=E\left(\beta_{u}(\tau)\right)<E(u)=c^{p o}$ for any $\tau \in\left([0,1] \backslash\left\{-s_{0} /\left(s_{1}-s_{0}\right)\right\}\right)$.

For every closed half-space $H$ define the path $\gamma_{u}^{H}:[0,1] \rightarrow \mathbb{R} \times S_{a}$ by $\gamma_{u}^{H}(\tau)=$ $\left(0,\left(\beta_{u}(\tau)\right)^{H}\right)$. By Lemma 4.2 and $\left\|u^{H}\right\|_{r}=\|u\|_{r}$ with $r \in[1, \infty)$, we have $\gamma_{u}^{H} \in$ $C\left([0,1], \mathbb{R} \times S_{a}\right)$. By Lemmas 4.2 and 4.3 , we obtain that $\tilde{E}\left(\gamma_{u}^{H}(\tau)\right) \leq \tilde{E}\left(\gamma_{u}(\tau)\right)$ for every $\tau \in[0,1]$ and then $\gamma_{u}^{H}(\tau) \in \Gamma$. Hence,

$$
\max _{\tau \in[0,1]} \tilde{E}\left(\gamma_{u}^{H}(\tau)\right) \geq c^{p o} .
$$

Since for every $\tau \in\left([0,1] \backslash\left\{-s_{0} /\left(s_{1}-s_{0}\right)\right\}\right)$,

$$
\tilde{E}\left(\gamma_{u}^{H}(\tau)\right) \leq \tilde{E}\left(\gamma_{u}(\tau)\right)<E(u)=c^{p o},
$$

we deduce that

$$
\tilde{E}\left(\gamma_{u}^{H}\left(\frac{-s_{0}}{s_{1}-s_{0}}\right)\right)=E\left(u^{H}\right)=c^{p o} .
$$

Hence $E\left(u^{H}\right)=E(u)$, which implies that

$$
\int_{\mathbb{R}^{N}}\left(I_{\alpha} *\left|u^{H}\right|^{p}\right)\left|u^{H}\right|^{p}=\int_{\mathbb{R}^{N}}\left(I_{\alpha} *|u|^{p}\right)|u|^{p} .
$$

By Lemma 4.3, we have $u^{H}=u$ or $u^{H}=u \circ \sigma_{H}$. By Lemma 4.4, we complete the proof.

Proof of Theorem 1.4. By Lemma 3.6 and Proposition 4.1, if $u \in \mathcal{G}$, then $|u| \in \mathcal{G}$ and $\|\nabla|u|\|_{2}=\|\nabla u\|_{2}$. Then we can proceed as Theorem 4.1 in [14, obtaining that

$$
\mathcal{G}=\left\{e^{i \theta}|v|: \theta \in \mathbb{R} \text { and }|v|>0 \text { in } \mathbb{R}^{N}\right\} .
$$

Moreover, by Proposition 4.5, the proof is complete.

Acknowledgements. This work is supported by the National Natural Science Foundation of China (No. 12001403).

\section{REFERENCES}

[1] T. Bartsch, Y. Liu, Z. Liu, Normalized solutions for a class of nonlinear Choquard equations, SN Partial Differ. Equ. Appl., (2020), 1-34.

[2] T. Bartsch, N. Soave, Multiple normalized solutions for a competing system of Schrödinger equations, Calc. Var. Partial Differ. Equ., 58 (2019), 22.

[3] J. Bellazzini, L. Jeanjean, T. Luo, Existence and instability of standing waves with prescribed norm for a class of Schrödinger-Poisson equations, Proc. London Math. Soc., 107 (2013), 303339.

[4] J. Bellazzini, G. Siciliano, Scaling properties of functionals and existence of constrained minimizers, J. Funct. Anal., 261 (2011), 2486-2507.

[5] H. Brezis, L. Nirenberg, Positive solutions of nonlinear elliptic equations involving critical Sobolev exponents, Commun. Pure Appl. Math., 36 (1983), 437-477.

[6] F. Brock, A. Yu. Solynin, An approach to symmetrization via polarization, Trans. Amer. Math. Soc., 352(4) (2000), 1759-1796. 
[7] T. Cazenave, P.L. Lions, Orbital stability of standing waves for some nonlinear Schrödinger equations, Commun. Math. Phys., 85 (1982), 549-561.

[8] S. Cingolani, L. Jeanjean, Stationary waves with prescribed $L^{2}$-norm for the planar Schrödinger-Poisson system, SIAM J. Math. Anal., 51(4) (2019), 3533-3568.

[9] B. Feng, X. Yuan, On the Cauchy problem for the Schrödinger-Hartree equation, Evol. Equ. Control Theory, 4 (2015), 431-445.

[10] F. Gao, M. Yang, On nonlocal Choquard equations with Hardy-Littlewood-Sobolev critical exponents, J. Math. Anal. Appl., 448(2) (2017), 1006-1041.

[11] F. Gao, M. Yang, On the Brezis-Nirenberg type critical problem for nonlinear Choquard equation, Sci. China Math., 61(7) (2018), 1219-1242.

[12] N. Ghoussoub, Duality and Perturbation Methods in Critical Point Theory, Cambridge Tracts in Mathematics, vol.107, Cambridge University Press, Cambridge, 1993, with appendices by David Robinson.

[13] E.P. Gross, Physics of many-Particle systems, Gordon Breach, New York, Vol.1, 1996.

[14] H. Hajaiej, C.A. Stuart, On the variational approach to the stability of standing waves for the nonlinear Schrödinger equation, Adv. Nonlinear Stud., 4(4) (2004), 469-501.

[15] L. Jeanjean, Existence of solutions with prescribed norm for semilinear elliptic equations, Nonlinear Anal., 28(10) (1997), 1633-1659.

[16] L. Jeanjean, K. Tanaka, A remark on least energy solutions in $R^{N}$, Proc. Amer. Math. Soc., 131(8) (2003), 2399-2408.

[17] L. Jeanjean, T.T. Le, Multiple normalized solutions for a Sobolev critical Schrödinger equation, arXiv: 2011.02945v1, 5 Nov 2020.

[18] X. Li, Studies of normalized solutions to Schrödinger equations with Sobolev critical exponent and combined nonlinearities, arXiv:2104.12997v2, 28 Apr 2021.

[19] X. Li, S. Ma, Choquard equations with critical nonlinearities, Commun. Contemp. Math., $22(04)(2020), 1950023$

[20] X. Li, S. Ma, G. Zhang, Existence and qualitative properties of solutions for Choquard equations with a local term, Nonlinear Anal.-RWA, 45 (2019), 1-25.

[21] G. Li, H. Ye, The existence of positive solutions with prescribed $L^{2}$-norm for nonlinear Choquard equations, J. Math. Phys., 55 (2014), 121501.

[22] E.H. Lieb, Existence and uniqueness of the minimizing solution of Choquard's nonlinear equation, Stud. Appl. Math., 57(2) (1977), 93-105.

[23] E.H. Lieb, M. Loss, Analysis, volume 14 of graduate studies in mathematics, American Mathematical Society, Providence, RI, (4) 2001.

[24] K. Liu, C. Shi, Existence of stable standing waves for the Schrödinger Choquard equation, Bound. Value Probl., (2018), 2018:160.

[25] X. Luo, Normalized standing waves for the Hartree equations, J. Differential Equations, 267 (2019), 4493-4524.

[26] H. Luo, Nontrivial solutions for nonlinear Schrödinger Choquard equations with critical exponents, Appl. Math. Lett., 107 (2020), 106422.

[27] V. Moroz, J. Van Schaftingen, Groundstates of nonlinear Choquard equations: existence, qualitative properties and decay asymptotics, J. Funct. Anal., 265(2) (2013), 153-184.

[28] V. Moroz, J. Van Schaftingen, Existence of groundstates for a class of nonlinear Choquard equations, Trans. Amer. Math. Soc., 367 (2015), 6557-6579.

[29] V. Moroz, J. Van Schaftingen, A guide to the Choquard equation, J. Fixed Point Theory Appl., 19(1) (2017), 773-813.

[30] S. Pekar, Untersuchung über die Elektronentheorie der Kristalle, Akademie Verlag, Berlin, 1954.

[31] R. Penrose, On gravity's role in quantum state reduction, Gen. Rel. Grav., 28 (1996), 581-600.

[32] M. Riesz, L'intégrale de Riemann-Liouville et le problème de Cauchy, Acta Math., 81 (1949), $1-223$.

[33] G. Siciliano, K. Silva, On the structure of the Nehari set associated to a Schrödinger-Poisson system with prescribed mass: old and new results, arXiv:2007.01718v1, 3 Jul 2020.

[34] N. Soave, Normalized ground states for the NLS equation with combined nonlinearities, J. Differential Equations, 269(9) (2020), 6941-6987.

[35] N. Soave, Normalized ground states for the NLS equation with combined nonlinearities: The Sobolev critical case, J. Funct. Anal., 279(6) (2020), 108610. 
[36] J. Van Schaftingen, M. Willem, Symmetry of solutions of semilinear elliptic problems, J. Eur. Math. Soc. (JEMS), 10(2) (2008), 439-456.

[37] J. Wei, Y. Wu, Normalized solutions for Schrödinger equations with critical Sobolev exponent and mixed nonlinearities, arXiv:2102.04030 $1,2021$.

[38] M.I. Weinstein, Nonlinear Schrödinger equations and sharp interpolation estimates, Comm. Math. Phys., 87 (1983), 567-576.

[39] M. Willem, Minimax Theorems, Birkhäuser, Boston, 1996.

[40] T. Yang, Normalized solutions for the fractional Schrödinger equation with a focusing nonlocal $L^{2}$-critical or $L^{2}$-supercritical perturbation, J. Math. Phys., 61 (2020), 051505.

[41] H. Ye, Mass minimizers and concentration for nonlinear Choquard equations in $R^{N}$, Topol. Methods Nonlinear Anal., 48 (2016), 393-417.

[42] S. Yuan, S. Chen, X. Tang, Normalized solutions for Choquard equations with general nonlinearities, Electron. Res. Arch., 28(1) (2020), 291-309.

XINFU LI

School of Science, Tianjin University of Commerce, Tianjin 300134, Peoples's Republic OF CHINA

Email address: lxylxf@tjcu.edu.cn 